F-actin distribution and function during sexual differentiation in Schizosaccharomyces pombe

Petersen, J; Nielsen, O; Egel, R; Hagan, I M; Nielsen, Olaf

Published in:

Journal of Cell Science

Publication date:

1998

Document version

Early version, also known as pre-print

Citation for published version (APA):

Petersen, J., Nielsen, O., Egel, R., Hagan, I. M., \& Nielsen, O. (1998). F-actin distribution and function during sexual differentiation in Schizosaccharomyces pombe. Journal of Cell Science, 111 (Pt 7), 867-76. 


\title{
F-actin distribution and function during sexual differentiation in
}

\section{Schizosaccharomyces pombe}

\author{
Janni Petersen ${ }^{1, *}$, Olaf Nielsen ${ }^{1}$, Richard Egel ${ }^{1}$ and lain M. Hagan ${ }^{2}$ \\ ${ }^{1}$ Department of Genetics, Institute of Molecular Biology, University of Copenhagen, DK-1353 Copenhagen K, Denmark \\ 2School of Biological Sciences, University of Manchester, 2.205 Stopford Building, Oxford Road, Manchester M13 9PT, UK \\ *Author for correspondence: jan0302@biobase.uk
}

Accepted 20 January; published on WWW 9 March 1998

\section{SUMMARY}

Sexual differentiation in Schizosaccharomyces pombe is induced from the $G_{1}$ phase of the cell cycle by nitrogen starvation and the presence of mating pheromones. We describe the distribution of $F$-actin during sexual differentiation. Cortical F-actin dots have previously been shown to be restricted to one end of the rod shaped cell during the $G_{1}$ phase of the cell cycle. Within half an hour of nitrogen starvation the distribution of cortical F-actin dots switched from being monopolar to bipolar. This was then reversed as the $\mathrm{F}$-actin cytoskeleton repolarized so that cortical F-actin dots accumulated towards the projection tip at one end of the cell. Following cell fusion, F-actin dots were randomly scattered during the horsetail movement that precedes meiosis I and remained scattered until prometaphase or metaphase of meiosis II, when they concentrated around the nucleus. F-actin was seen on the lagging face of the nuclei which faced the partner nucleus during anaphase B of meiosis II. Early on in this anaphase F-actin was also seen on the opposite side of the nucleus, near the spindle pole body. F-actin accumulated within the spores in the mature ascus. Treatment with the actin depolymerising drug Latrunculin A showed that F-actin is required for cell fusion and spore formation. Latrunculin A treatment extended all stages from karyogamy to meiosis I. The $S$. pombe homologue of the actin binding protein profilin, Cdc3, was shown to be required for conjugation. Cdc3 co-localized with the formin related molecule Fus1 at the projection tip. The polarization of $\mathbf{F}$-actin cortical dots to the projection tip was unaffected in the $c d c 3.124$ mutant, but $c d c 3.124$ mutant cells were unable to break down the cell walls between the two cells following agglutination.

Key words: Schizosaccharomyces pombe, Profilin, Actin, Latrunculin A, Conjugation, Meiosis

\section{INTRODUCTION}

The actin cytoskeleton plays a key role in the establishment and maintenance of polarized growth and the execution of cell division of the yeasts Saccharomyces cerevisiae and Schizosaccharomyces pombe (reviewed by Bretscher et al., 1994; Robinow and Hyams, 1989). Progress through the cell cycle is accompanied by rearrangement and polarization of cytoplasmic microfilaments. There are two types of actin filaments in yeasts, cytoplasmic cables and cortical dots (Kilmartin and Adams, 1984; Marks and Hyams, 1985). As in fungi in general, cortical dots cluster at the growing tip and at the cytokinetic ring, whilst cables extend from the tip towards the main body of the cell (Heath, 1990). In Saccharomyces cerevisiae, it has been demonstrated that cortical F-actin dots are motile, responding rapidly to external stimuli (Waddle et al., 1996; Doyle and Botstein, 1996). Recent studies have shown that there are at least two molecular pathways for the establishment of polarity in budding yeast; one which requires actin and one which does not (Ayscough et al., 1997). The actin dependent pathway localizes secretory vesicles to facilitate polarized cell surface growth.

Growth of $S$. pombe cells occurs by tip extension which follows a defined pattern under logarithmic growth conditions in minimal media (Mitchison and Nurse, 1985). Immediately after cell division, growth is restricted to the tip which existed before division (the old end). After a third of the total duration of the cell cycle when a critical cell length has been obtained, the tip which was formed by cytokinesis (the new end) starts growing. This process is referred to as new end take off (NETO). After NETO both tips elongate until the critical cell size for division is attained whereupon growth stops and cell division is initiated. The distribution of cortical F-actin dots mirrors these growth patterns (Marks and Hyams, 1985). The dots concentrate wherever cell extension is occurring until growth is switched off as division is initiated. At this time Factin leaves the tips and a ring is formed in the middle of the cell. The F-actin ring appears to contract and is replaced by an array of dots on either side of the septum once cytokinesis is complete.

The polymerization state of actin is influenced by interactions with a number of actin binding proteins (Mullins et al., 1996). Profilin is an actin binding protein found in all eukaryotes examined to date (Sohn and GoldschmidtClermont, 1994). It was initially identified in a stable 1:1 protein complex with actin monomers (G-actin) (Carlsson et 
al., 1976). It has been suggested that the function of profilin is to sequester actin monomers and so prevent polymerization of G-actin into filaments (F-actin) (Carlsson et al., 1977). However, a number of studies have shown that the role played by profilin in regulating actin dynamics is more complex. Profilin both inhibits and promotes actin polymerization (reviewed by Sohn and Goldschmidt-Clermont, 1994). It is involved in growth factor-mediated signal transduction to the actin cytoskeleton, and binds the membrane bound phospholipid PIP2. Profilin has also been shown to interact with proline rich regions by a number of different approaches. In $S$. pombe the $c d c 3^{+}$gene encodes a profilin homologue (Balasubramanian et al., 1994). Cdc3 function is required for the formation of the actin contractile ring but not for tip growth. Deletion, or overexpression of profilin results in an inability to form the cytokinetic ring and cells fail to execute cytokinesis (Balasubramanian et al., 1994). In S. cerevisiae disruption of the profilin encoding PFY1 gene results in morphological defects (Haarer et al., 1990).

Mating in $S$. pombe is accompanied by a series of elaborate morphological changes. Upon nitrogen starvation diffusible mating pheromones induce polarized cell growth of cells of the opposite mating types towards one another. Upon contact, cells agglutinate at the projection tips, and localized cell wall degradation between the two partners results in cell fusion enabling karyogamy. After karyogamy the resulting diploid zygote enters meiosis and sporulates (reviewed by Nielsen and Davey, 1995).

We describe the changes in the distribution of F-actin that accompany sexual differentiation, show that depolymerization of F-actin blocks conjugation and spore wall formation and demonstrate a requirement for profilin during conjugation.

\section{MATERIALS AND METHODS}

\section{Strains, media and genetic methods}

The $S$. pombe strains used are listed in Table 1. Cells were grown in either MSL or MSL-N (minimal sporulating liquid/agar) (Egel et al., 1994). Standard classical and molecular genetic techniques for $S$. pombe were used as described previously (Gutz et al., 1974; Moreno et al., 1991). Because cells commit to conjugation following one or two division cycles, immediately shifting $c d c 3.124$ cells to the restrictive temperature would result in a defective cytokinesis which would obscure any effects of the mutation upon conjugation. Therefore the following quantitative mating assay was adopted: the two heterothallic $c d c 3.124$ ts $^{-}$strains of opposite mating types were grown in MSL to a density of $5 \times 10^{6}$ cells $\mathrm{ml}^{-1}$ at the permissive temperature of $25^{\circ} \mathrm{C}$, washed in MSL-N (lacking nitrogen) and resuspended in MSL-N at $5 \times 10^{6}$ cells $\mathrm{ml}^{-1}$. Following incubation at

Table 1. S. pombe strains used in this study

\begin{tabular}{lll}
\hline Strain & \multicolumn{1}{c}{ Genotype } & \multicolumn{1}{c}{ Source or reference } \\
\hline EG 282 & $h^{90}$ & Nielsen and Egel, 1990 \\
EG 325 & $h^{90}$ ura4-D18 & Nielsen et al., 1992 \\
EG 544 & $h$ - mat2,3::LEU2 & Egel et al., 1994 \\
EG 545 & $h^{+}$mat $2,3: \because$ LEU2 & Nielsen et al., 1992 \\
EG 919 & $h^{-}$cdc3.124 & This study* \\
EG 920 & $h^{+}$mat2,3::LEU2 cdc3.124 & This study \\
EG 1001 & $h^{90}$ cdc10.v50 & This study $\dagger$ \\
IHSP1081 & $h^{90}$ cut12-GFP leu1.32 ura4-D18 & Bridge et al., 1998
\end{tabular}

*The $c d c 3.124$ allele was described by Balasubramanian et al. (1994). $\dagger$ The cdc10.v50 allele was described by Marks et al. (1992). the permissive temperature for 2.5 hours the two populations were mixed in a 1:1 ratio and shifted to the restrictive temperature of $32^{\circ} \mathrm{C}$. The efficiency of mating was calculated according to the method of Petersen et al. (1995).

\section{Molecular manipulations}

Standard procedures for bacterial and DNA manipulation were carried out according to the method of Sambrook et al. (1989). E. coli DH5 (Hanahan 1985) was used for propagation of plasmids. PCR amplification was carried out in $50 \mu \mathrm{l}$ reaction mixtures (Kocher et al., 1989). Generation of a version of $c d c 3$ containing three copies of the hemagluttinin epitope tag recognised by the 12CA5 antibody at its amino terminus was as follows: the ORF of $c d c 3$ amplified by PCR using two $c d c 3^{+}$specific primers JPP29: 5'CGCGTCGACATATGTCTTGGCAAGCTTACGTT and JPP32: 5'CGCAGATCTAATACCCAACACCAACAA and a cDNA library as template (kindly provided by Chris Norbury) was cloned in frame into the NdeI and BamHI site of pREP41HAN (Griffiths et al., 1995). This tagged $c d c 3$ construct was able to complement the temperature sensitive growth defect of $c d c 3.124$ at $36^{\circ} \mathrm{C}$.

\section{Fluorescence microscopy}

For fluorescence microscopy of conjugating cells, cells were grown in MSL to a density of $5 \times 10^{6}$, washed in MSL-N and starved in MSL$\mathrm{N}$ for 5 hours. Rhodamine conjugated phalloidin was used to visualize F-actin according to the procedures of Marks and Hyams (1985). Digestion in $250 \mu \mathrm{g} / \mathrm{ml}$ zymolyase for 60 minutes following fixation was required to visualize the F-actin cytoskeleton in the later stages of meiosis. Affinity purified anti-Cdc3 antibodies (Balasubramanian et al., 1994) or tissue culture supernatant containing 12CA5 monoclonal antibodies (Wilson et al., 1984; Meloche et al., 1992) were used to visualize $\mathrm{Cdc} 3$, or epitope tagged $c d c 3$, respectively, following combined formaldehyde and glutaraldehyde fixation (Hagan and Hyams, 1988) in PM buffer (Marks and Hyams, 1985). Image processing was done according to the method of Hagan and Yanagida (1995).

\section{Latrunculin A treatment}

$200 \mu \mathrm{l}$ of cells were removed from the culture and Latrunculin A (a generous gift from Dr K. Ayscough) was added to a final concentration of $10 \mu \mathrm{M}$ from a $200 \mu \mathrm{M}$ stock solution in DMSO. Mock treatment with an equal volume of DMSO did not affect sexual differentiation (data not shown). The $c d c 10 . v 50$ synchronized mating was brought down to $30^{\circ} \mathrm{C}$ at medium shift.

\section{RESULTS}

\section{Pheromone induced polarization of S. pombe cells is accompanied by polarization of the F-actin cytoskeleton}

In the absence of nitrogen, $S$. pombe cells pause in $\mathrm{G}_{1}$ and either enter stationary phase or commit to sexual differentiation. The presence of pheromone from a cell of the opposite mating type induces sexual differentiation, which includes pheromone-induced polarization, conjugation and sporulation (reviewed in Nielsen and Davey, 1995). To examine the rearrangements of the F-actin cytoskeleton that accompany sexual differentiation, a homothallic $h^{90}$ strain was starved of nitrogen and cells were stained with rhodamineconjugated phalloidin. Cortical F-actin dots concentrated at the projection tip during pheromone-induced polarization and the initial stages of cell fusion (Fig. 1A). Immediately following cell wall degradation and karyogamy the cell wall expands at the fusion point in order to form the zygote. F-actin was still 
Fig. 1. F-actin localization during pheromone induced sexual differentiation in fission yeast. The Fig. shows a series of paired panels, each pair showing rhodaminephalloidin staining (top) and chromatin DAPI/phase contrast images (bottom) of the same cells. F-actin localization in wild-type cells shows that F-actin cortical dots were concentrated at the projection tip during pheromone induced polarization and fusion (AB). Following fusion F-actin was seen as dispersed cytoplasmic dots during horsetail movement $(C)$ and meiosis I (D). After meiosis I and the beginning of meiosis II F-actin concentrated around the chromatin (E-F). (F) In early anaphase B of meiosis II F-actin was seen both as a small cap on the leading side of the nuclei at a position which roughly equated with the position of the SPB (arrowheads) and as larger aggregates on the opposite lagging side of the nuclei (small arrows). (G) In telophase of meiosis II F-actin was only localized at the side of the nuclei facing the partner from meiosis II. (H) In mature asci, where the spore wall has been formed, F-actin concentrates within the spores and is excluded from the surrounding cytoplasm. Bar, $5 \mu \mathrm{m}$.
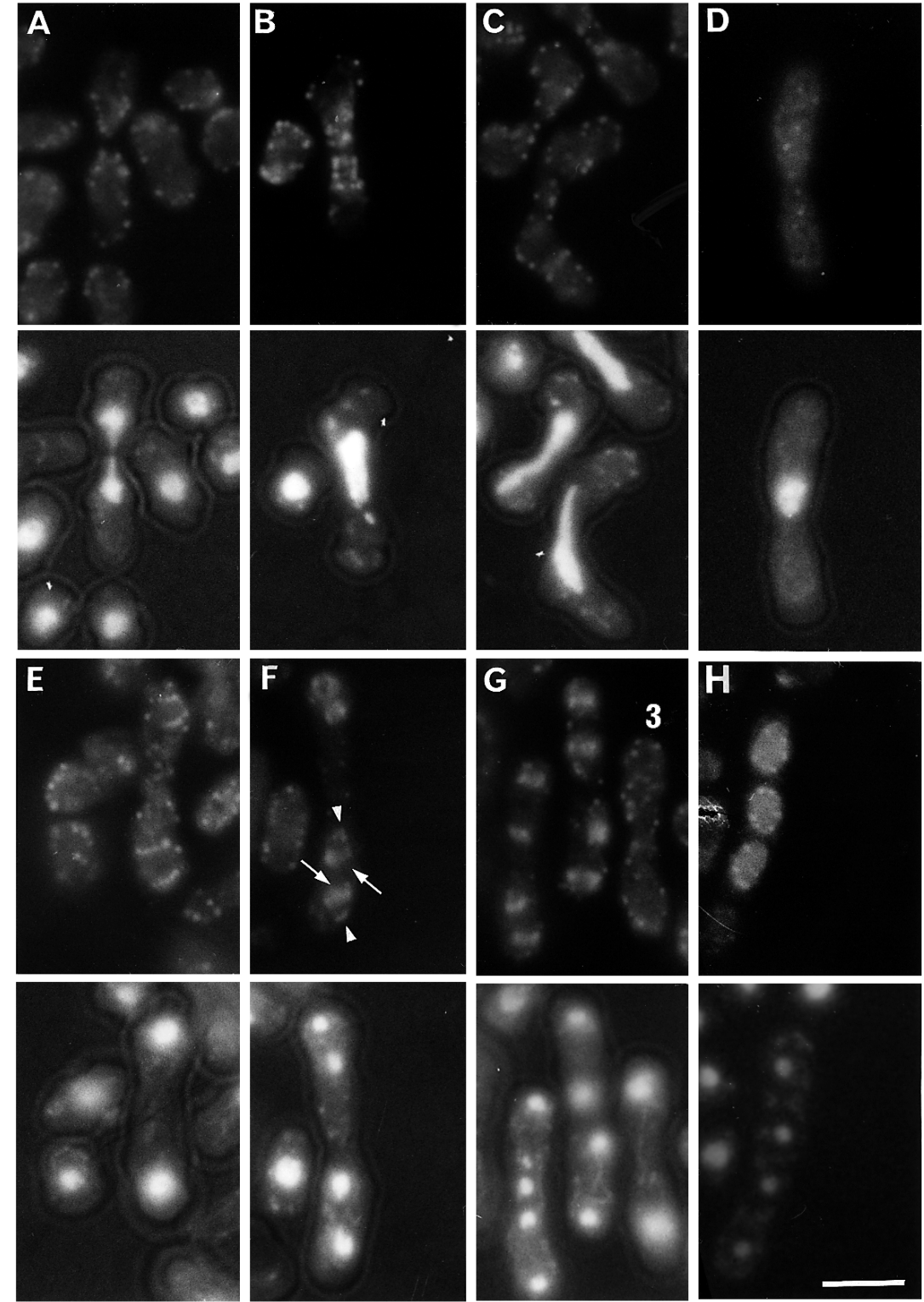

concentrated in the central region of the zygotes during this process (Fig. 1B). It is important to note that whilst F-actin accumulates in this region before and after fusion, F-actin staining was absent from the precise region where fusion occurs. We conclude that during conjugation F-actin distribution correlates with sites of polarized cell growth.

\section{F-actin distribution during meiosis}

In a wild-type strain of $S$. pombe the diploid zygotes enter meiosis immediately (Egel, 1973). The first stage of meiosis is a period of vivid nuclear migration which is referred to as 'horsetail movement' because of the morphology assumed by the nucleus (Robinow, 1977; Chikashige et al., 1994). F-actin dots were scattered throughout the cytoplasm during horsetail movement (Fig. 1C). F-actin dots remained scattered until two chromatin masses had been separated to either end of the cell (Fig. 1D,G cell 3). Some of the nuclei at this stage were surrounded by $\mathrm{F}$ actin dots (Figs 1E, 2A). In early anaphase B of meiosis II F-actin was seen both as a small cap on the leading side of the nuclei at a position which roughly equated with the position of the spindle pole body (SPB) (arrowheads Figs $1 \mathrm{~F}, 2 \mathrm{~B}$ ) and as larger aggregates on the opposite lagging side of the nuclei (small arrows Figs 1F, 2B). Later, F-actin was seen only on the lagging side of the nuclei facing the partner nucleus (Figs 1G, 2C). In mature asci, where the spore wall has been formed, F-actin dots were seen within the spore but not in the surrounding cytoplasm (Fig. 1H, 2D). Thus, again, F-actin distribution correlates with sites of cell wall deposition.

Because it was not possible to determine whether the nuclei around which F-actin first accumulated were in meiosis I or meiosis II from the morphology of the chromatin alone we stained a strain in which the SPBs had been marked with a Cut12-GFP fusion protein (Bridge et al., 1998). Two SPBs on a single nucleus indicates that the nucleus is in meiosis II (Hagan and Yanagida, 1995). Clustering of F-actin around the nuclei was only seen in cells with two SPBs and then only when both SPBs were on either side of the chromatin indicating that it occurs at pro-metaphase or metaphase of meiosis II (Fig. 3). 


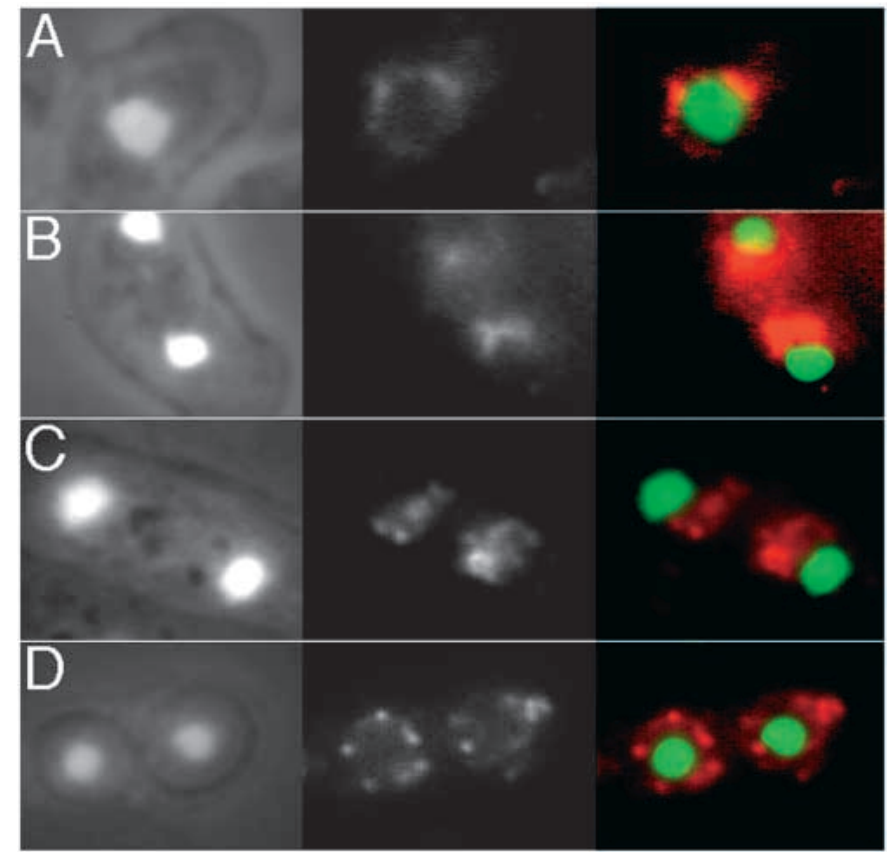

Fig. 2. Superimposition of F-actin and chromatin during meiosis II. The figure shows four sets of panels, each set shows the following images of the same cells: DAPI/phase contrast - left, phalloidin centre, and a combined phalloidin (red) and DAPI (green) - right. (A) F-actin accumulated around the chromatin. (B) In early anaphase $\mathrm{B}$ of meiosis II F-actin was seen both as a small cap on the leading side of the nuclei at a position which roughly equated with the position of the SPB and as larger aggregates on the opposite lagging side of the nuclei. (C) Later F-actin only localized at the side of the nuclei facing the partner nucleus. (D) In mature asci F-actin concentrated within the spores and is excluded from the surrounding cytoplasm.

\section{Nitrogen starvation induced depolarization of the F- actin cytoskeleton before it re-polarized at the projection tip}

We sought conditions in which it would be possible to study the rearrangement of the actin cytoskeleton in cells which are committed to mating but are yet to conjugate. We reasoned that if cell-cycle progression was arrested in a $\mathrm{G}_{1}$ state before starvation, an entire population could be followed as they synchronously conjugated once the nitrogen source was removed. To this end we used the temperature-sensitive mutant cdc10.v50 (Marks et al., 1992). cdc10 mutants arrest prior to START in $\mathrm{G}_{1}$ at the restrictive temperature in a state competent to conjugate (Nurse et al., 1976, Nurse and Bissett 1981). An $h 90$ cdc10.v50 culture was arrested for 4 hours at the restrictive temperature of $36^{\circ} \mathrm{C}$ to accumulate in $\mathrm{G}_{1}$ and simultaneously shifted to $32^{\circ} \mathrm{C}$ and inoculated into nitrogen free medium. This treatment enabled the cells to start sexual differentiation much faster than wild-type cells (Fig. 4). It was necessary to bring the temperature down to $32^{\circ} \mathrm{C}$ because above this temperature conjugation is inhibited. Prior to conjugation in liquid media, cells must agglutinate in order to bring the two mating partners together. Agglutination is one of the first signs of sexual activity (Egel, 1971). In wild-type cells, agglutination was observed after 3.5 hours, whereas agglutination occurred within less than an hour of starvation of the $h^{90} c d c 10 . v 50$

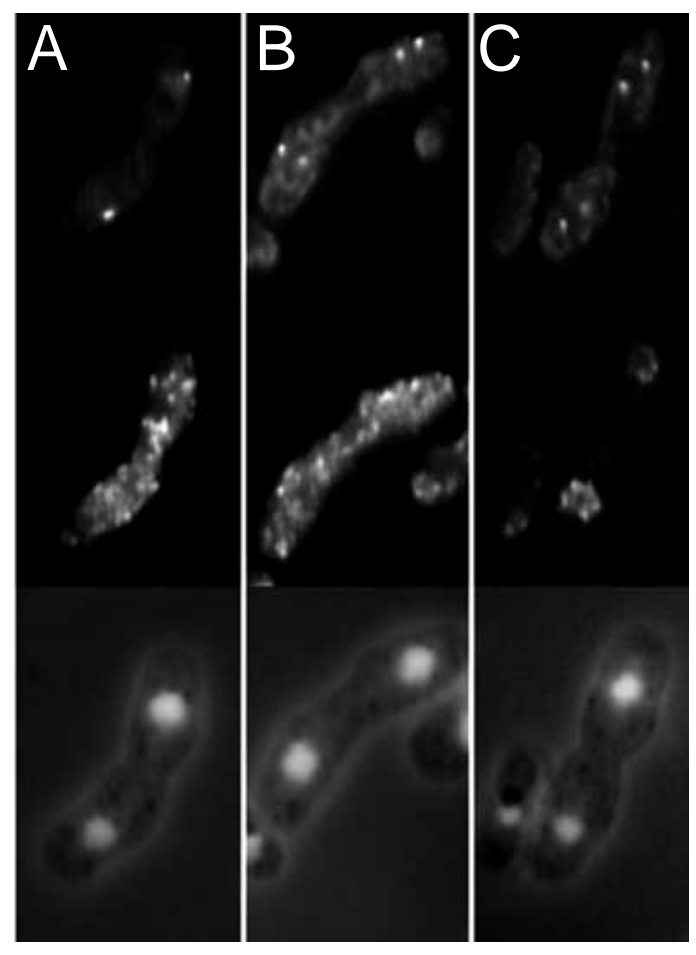

Fig. 3. F-actin accumulation around the meiotic nuclei occurs in prometaphase or metaphase of meiosis II. The Fig. shows three separate figures. In each case the left-hand panel shows the SPBs marked with a Cut12-GFP fusion protein the middle panel shows phalloidin staining, and the right-hand panel shows the chromatin DAPI/phase contrast images of the same cells. (A) Throughout meiosis I F-actin dots were scattered throughout the cytoplasm. (B) In prophase of meiosis II when the SPBs duplicated F-actin was still scattered. Whereas, in prometaphase or metaphase (C) when the SPBs were on either side of the chromatin (Hagan and Yanagida, 1995), F-actin accumulated around the nuclei.

strain (data not shown). At the restrictive temperature the $h^{-}$ $c d c 10.129$ mutant arrests vegetative cell growth with F-actin only present at one end (Marks et al., 1987). Similarly, phalloidin staining of the $h^{90}$ cdc10.v50 strain showed that, before nitrogen starvation, $73 \%$ of the cells had a monopolar distribution of F-actin (Fig. 5A). Within half an hour of nitrogen starvation (Fig. 5B) F-actin became tightly associated with both ends of the cell (after half an hour only 4\% of the cells showed F-actin polarization to only one end). Cortical Factin dots were still seen at both ends of the cell 1 hour after medium shift (F-actin was monopolar in only 6\%), but after 1.5 hours of nitrogen starvation $29 \%$ of the cells once again showed polarization of F-actin to one end (Fig. 5D). 20\% of the cells had fused after 2 hours and F-actin remained localized to the point of fusion (Fig. 5E). Thus, F-actin localization in the cdc10.v50 strain resembled that of wild type at the projection tip and the point of fusion, but enabled a better understanding of the response of $\mathrm{G}_{1}$ cells to starvation and pheromone. The kinetics of the mating response of the cdc10.v50 strain differs from wild type (Fig. 4) because wildtype cells continue to divide and stay attached to their sister cell which often has the opposite mating type (Miyata and Miyata, 1981). Therefore the chance of finding a mating 


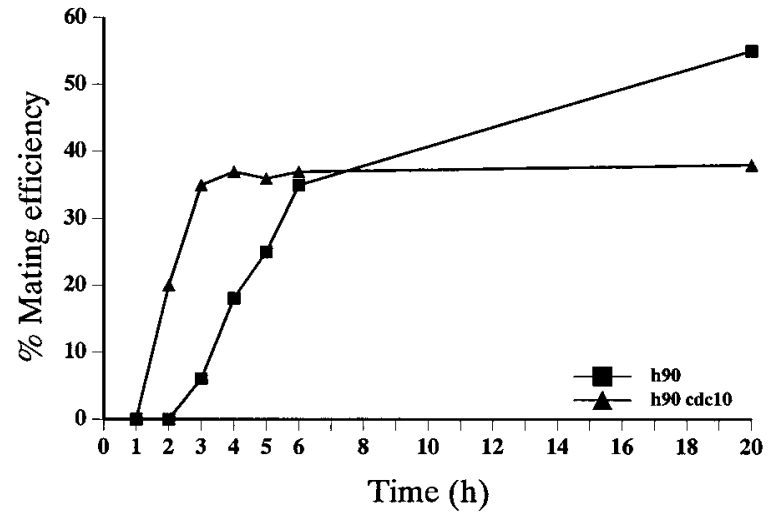

Fig. 4. Synchronization of conjugation in S. pombe. An $h^{90} c d c 10$ $v 50$ culture was treated as described in the text. The timing of conjugation was compared with a wild-type $h^{90}$ culture. In the experiment shown in this figure sexual agglutination was seen in less than 1 hour in the $h^{90} c d c 10$-v50 culture (not shown) and the first cells initiate mating within 1.5 hours. In contrast, in the wild-type control culture, agglutination was observed after 3.5 hours but $5 \%$ of the cells had started conjugation before agglutination was visible. This conjugation is presumably sister cell conjugation (see text). Importantly, mating is completed more rapidly in the $c d c 10-v 50$ synchronized mating indicating that within the population conjugation from this $\mathrm{G}_{1}$ arrest was more synchronous than in the wild-type culture. Whilst the exact timing of the initiation of conjugation varies by around 15 minutes between different experiments (see for example Fig. 7), wild-type cells always show a similar delay in conjugation when compared to the mutant strain.

partner during agglutination is significantly higher for wildtype cells and more cells eventually mate. We shall refer to cdc10.v50 cultures which undergo sexual differentiation from the $\mathrm{G}_{1}$ phase of the cell cycle as a 'cdc10.v50 synchronized mating'.

\section{Depolymerization of actin filaments with Latrunculin A blocks conjugation, spore wall formation and affects meiotic progression}

In order to examine the role of F-actin during sexual differentiation, cells were treated with the drug Latrunculin A (LAT A) which depolymerizes actin in different organisms including S. cerevisiae (Ayscough et al., 1997). F-actin distribution was similarly affected in S. pombe (Fig. 6A-D) showing that LAT A also disrupts actin filaments in fission yeast.

When LAT A was added to a cdc10.v50 synchronized mating in which the cells had already initiated, agglutination fusion and projection tip formation were both inhibited (Fig. $6 \mathrm{E}, \mathrm{F})$. No zygotes were seen four hours after drug addition whereas $31 \%$ of the cells in the control culture treated with DMSO alone had fused and many now had horsetail nuclei (Fig. 6F, Table 2).

LAT A was added to a cdc10.v50 synchronized mating 4, 6 and 8 hours after starvation in order to examine actin function during meiosis. DAPI staining was used to score zygotes according to the nuclear morphologies indicated in Table 2. As conjugation was complete within 4 hours of starvation (Fig. 4) any changes in the relative percentages of different classes represented effects on progression through meiosis.

Data from the aliquot to which LAT A was added 4 hours
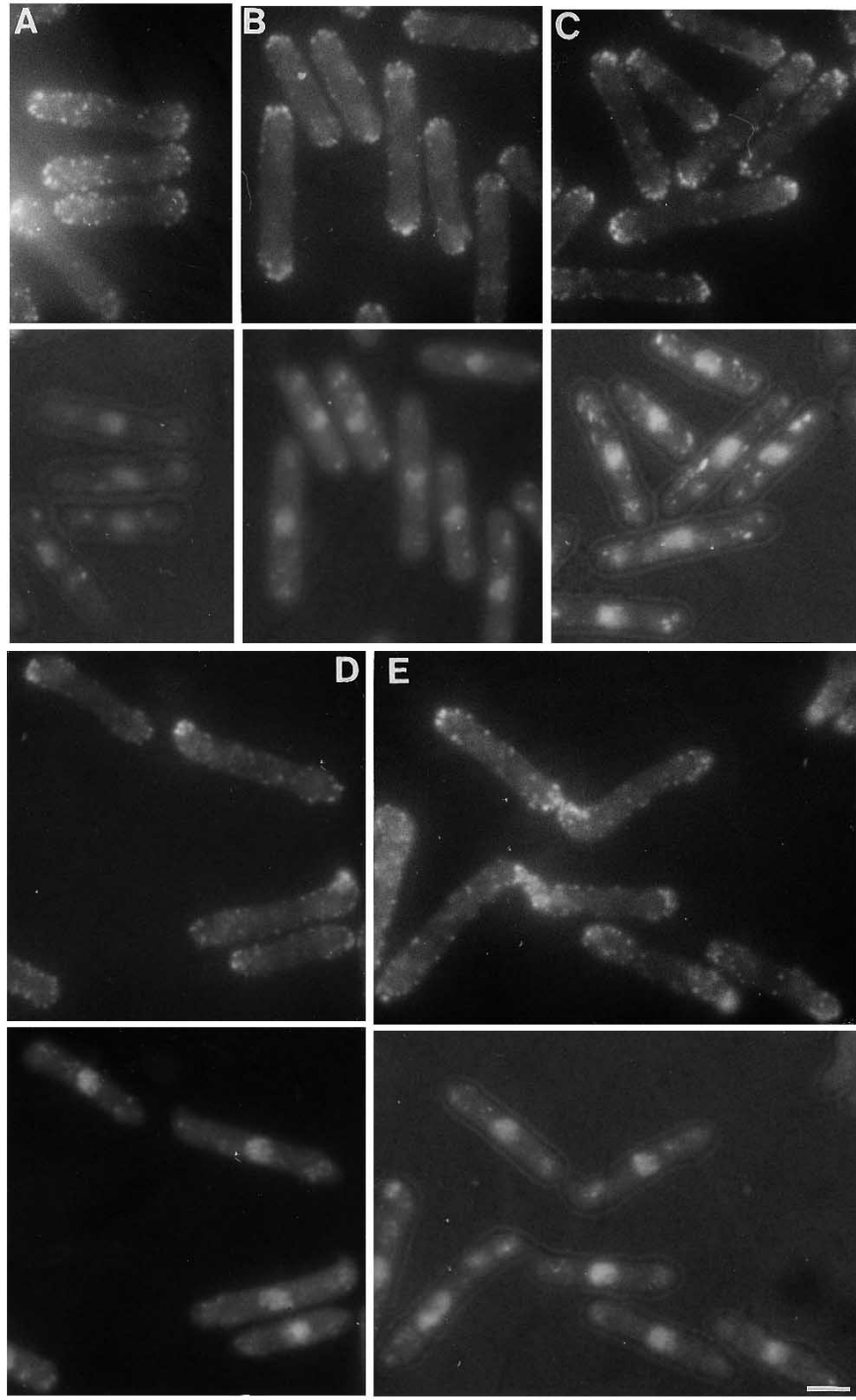

Fig. 5. Nitrogen starvation induces depolarization of the F-actin cytoskeleton prior to polarization at the site of conjugation in a cdc10-v50 synchronised mating. The figure shows a series of paired panels, phalloidin (top) and DAPI/phase contrast images (bottom) of the same cells. (A) Before nitrogen starvation the F-actin localization was monopolar in the $\mathrm{G}_{1}$ arrested $h^{90} c d c 10-v 50$ strain (Marks et al., 1987). The tip association in cdc10.v50 was not particularly tight, but the out of focus signals clearly make one tip brighter indicating monopolarity. The tighter tip association reported by Marks et al. (1987) was presumably because the cells were only shifted to the restrictive temperature for 6 hours in their study as opposed to the 4 hours we have used here. (B) After 0.5 hour of nitrogen starvation the F-actin localization was bipolar. (C) Bipolar localization of Factin was maintained after 1 hour of nitrogen starvation when agglutination occurred. (D) monopolar F-actin localization was reestablished when the projection tips started forming after 1.5 hours. (E) After 2 hours fusion had occurred in $20 \%$ of the cells and F-actin remained localized in the fusion region. Bar, $5 \mu \mathrm{m}$.

after induction of sexual differentiation by medium shift were most informative. A drop in the percentage of zygotes with 2 nuclei from $39 \%$ at 4 hours to $2 \%$ at 6 hours was seen when LAT A was added. This indicated that the 4 hour binucleate zygotes are yet to do karyogamy and that drug treatment does 


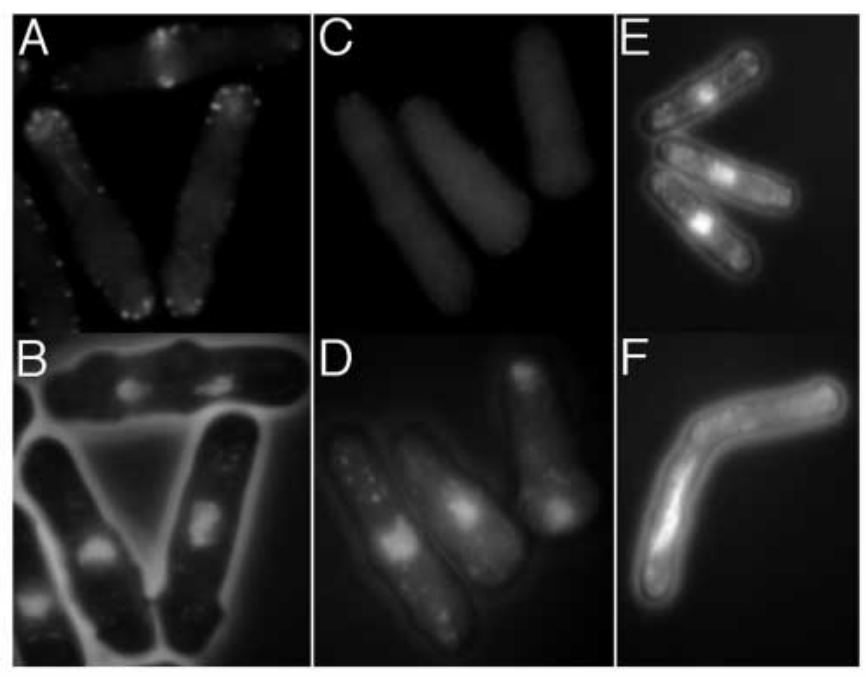

Fig. 6. Latrunculin A treatment depolymerizes actin filaments and blocks conjugation. (A-D) F-actin localisation in cells stained with rhodamine-conjugated phalloidin and DAPI before (A,B) and after (C,D) treatment with $200 \mu \mathrm{M}$ LAT A. (A and C) Phalloidin staining; (C and D) DAPI/phase contrast images of the same cells. F-actin is completely depolymerized within ten minutes of drug addition (C). (E,F) cdc10.v50 $\mathrm{h}^{90}$ cells were synchronized for conjugation as in Fig. 4 and treated with Latrunculin A 1.5 hours after starvation. The panels show DAPI/phase contrast images after a further 2.5 hours incubation either in the presence $(\mathrm{E})$ or absence $(\mathrm{F})$ of the drug.

not block karyogamy. Cells rapidly committed to the horsetail stage in the absence of the drug (indicated by the low frequency of zygotes with single nuclei after 4 and 6 hours). In contrast, we conclude that drug treatment resulted in accumulation of cells prior to the horsetail stage. This conclusion is drawn because the number of cells with a horsetail at 6 hours was $54 \%$ as compared to $74 \%$ in the control culture and because $40 \%$ of the cells in the drug treated culture had 1 nucleus (which is consistent with 39\% of the zygotes being at a stage prior to karyogamy at 4 hours). The final conclusion from the data set generated by drug addition at 4 hours was that drug treatment blocked spore formation. 8 hours after medium shift $32 \%$ of the control zygotes had 4 nuclei and none had spores walls. As these figures had changed at 22 hours to $9 \%$ and $75 \%$ respectively, all of the zygotes with 4 nuclei and no spore walls at 8 hours had formed spore walls by 22 hours. This contrasted with only $1 \%$ of the population having spores at 22 hours if LAT A had been added, despite the fact that $13 \%$ had four nuclei at 8 hours. Thus, these $13 \%$ were unable to form spore walls.

The conclusion that actin was required for spore wall formation was confirmed by data from other time points. There was remarkable agreement between the number of zygotes with 4 nuclei at the time of drug addition and the proportion of asci at the end of the experiment. This means that the requirement for actin for spore wall formation is complete by the time 4 nuclei are separated.

The final conclusion from the experiment comes from the aliquot to which the drug was added 6 hours after medium shift. Drug treatment slows down exit from the horsetail stage and cells accumulate before nuclear separation in meiosis I.
Table 2. The effect of treatment with LAT A on meiotic progression

\begin{tabular}{lccccc}
\hline $\begin{array}{l}\text { Time since } \\
\text { starvation } \\
\text { (hours) }\end{array}$ & Horsetail & 1 nucleus & 2 nuclei & $\begin{array}{c}\text { 4 nuclei } \\
\text { (no spores) }\end{array}$ & Spores \\
\hline $\begin{array}{c}\text { No LAT A } \\
4\end{array}$ & 52 & 6 & 39 & 3 & 0 \\
6 & 74 & 4 & 15 & 7 & 0 \\
8 & 25 & 22 & 21 & 32 & 0 \\
22 & 5 & 1 & 10 & 9 & 75 \\
LAT A & & & & & \\
4 & 54 & 40 & 2 & 4 & 0 \\
6 & 52 & 28 & 7 & 13 & 0 \\
8 & 11 & 48 & 13 & 27 & 1 \\
22 & 36 & 40 & 10 & 14 & 0 \\
LAT A & & & & & \\
6 & 6 & 32 & 19 & 36 & 7 \\
8 & & & & & \\
22 & 0 & 10 & 12 & 45 & 33 \\
LAT A & & & & & \\
8 & & & & &
\end{tabular}

Latrunculin A (LAT A) was added to a $c d c 10 . v 50$ synchronized mating at the times following nitrogen starvation which are indicated in the left hand column of the table in italics. The percentage of zygotes in which the nuclei had the morphologies indicated at the top of each column were scored at the times indicated in the left-hand column. For each time point $n=150$.

\section{The S. pombe profilin homolog, Cdc3, is essential for conjugation}

We next sought a genetic approach to disrupting actin function during conjugation. Whilst we are unaware of strong conditional mutations in the actin gene $a c t 1^{+}$itself (Mertins and Gallwitz, 1987), several conditional mutants exist in molecules which modulate actin function. Profilin was selected as the protein for study because it regulates actin dynamics in diverse systems and is thought to interact with proteins of the formin family (Evangelista et al., 1997; Jansen et al., 1996; Chang et al., 1997; Imamura et al., 1997). We have previously shown that the fission yeast formin Fus1 is required for conjugation suggesting that profilin too may be involved in this process (Petersen et al., 1995). In the fus $1 . B 20$ mutant and cells completely lacking the fus 1 gene product cytoplasmic fusion is prevented as the cell walls separating the mating partners are not degraded.

We measured the ability of the $c d c 3.124$ ts $^{-}$mutant to mate at a temperature which is restrictive for the mitotic function of $c d c 3.124$ under the conditions used, $32^{\circ} \mathrm{C}$. All of the 200 cells attempting to conjugate that we examined displayed a fus ${ }^{-}$ phenotype (Fig. 7B). Calcofluor staining showed that the cell wall was still intact between these prezygotes (Fig. 7A). We have previously shown that only one of the two partners needs to have Fus1 activity for cells to fuse although the efficiency is reduced (Petersen et al., 1995). Similarly, we found that if wild-type and $c d c 3.124$ cells were mated in liquid cultures 5\% of cells conjugated, whereas if both strains were wild-type, $19 \%$ cells conjugated. These data clearly show that provision of $\mathrm{Cdc} 3$ activity from one partner is sufficient to break down the separating cell walls, albeit at a reduced efficiency. Thus, the fus ${ }^{-}$phenotype of $c d c 3.124$ strongly resembles that of fus 1.B20 mutant cells. 

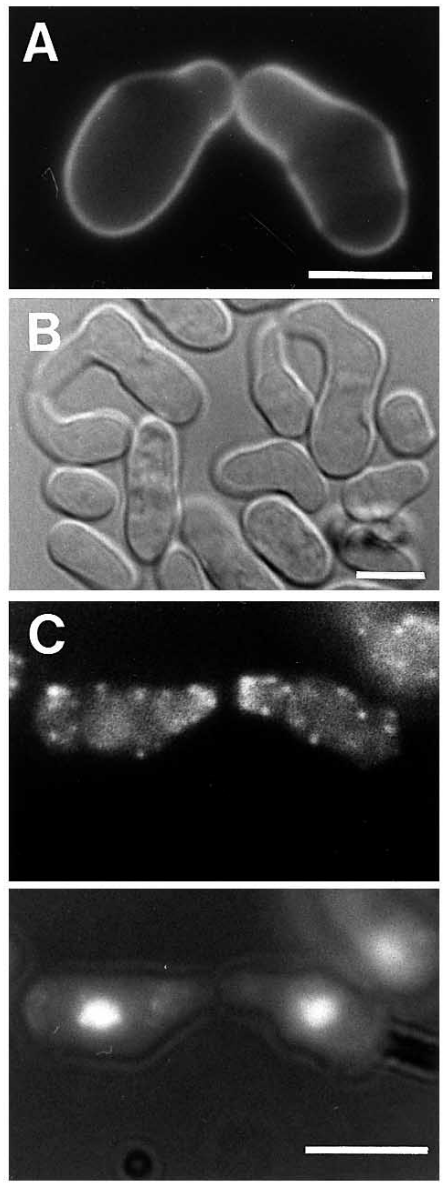

\section{F-actin localization to the projection tip is unaffected by the $c d c 3.124$ mutation during conjugation}

In fission yeast profilin is required for the formation of the actin contractile ring. Deletion or overexpression of $\mathrm{Cdc} 3$ results in an inability to form the actin ring (Balasubramanian et al., 1994). Thus, the observed fus ${ }^{-}$phenotype in the $c d c 3.124$
Fig. 7. Cdc3, an $S$. pombe profilin homolog is essential for conjugation. (B) The $c d c 3.124 \mathrm{ts}^{-}$mutant fails to conjugate at $32^{\circ} \mathrm{C}$ in liquid culture, as cell blocked with a fus ${ }^{-}$phenotype. (A) Staining with calcofluor shows that the cell wall is still intact between these prezygotes. (C) Phalloidin staining shows that F-actin dots are still polarized in the $c d c 3.124$ mutant, indicating that $\mathrm{Cdc} 3$ is not required for F-actin polarization during conjugation. A DAPI/phase contrast image of the same cells is shown below. Bar, $5 \mu \mathrm{m}$.

mutant could be due to an inability of $\mathrm{Cdc} 3$ to recruit F-actin to the tip. We therefore determined whether the $c d c 3.124$ mutant was capable of locating F-actin during mating. F-actin dots were still present at the projection tip at the restrictive temperature (Fig. 7C) indicating that, at the level of fluorescence microscopy, $\mathrm{Cdc} 3$ is not required to direct the localization of F-actin during mating.

\section{Cdc3 localizes to the tip in conjugating cells}

In wild-type $\mathrm{Cdc} 3$ localization resembled that of Fus 1: cytoplasmic dot staining prior to conjugation was followed by $\mathrm{Cdc} 3$ association with the projection tip and the final reappearance of cytoplasmic dots after conjugation (Fig. 8AB). An identical localization pattern was observed with a functional $\mathrm{Cdc} 3$ fusion protein tagged with three copies of the HA epitope at its amino terminus (Fig. 8C). A Fus1-GFP fusion localized to the projection tip (J. Petersen et al., unpublished) and simultaneous localization of Cdc3 and Fus1GFP in the same cell showed that the two proteins co-localize at the projection tip (Fig. 8D). Thus, the localization of $\mathrm{Cdc} 3$ to the projection tips during conjugation supported the finding that it is essential for conjugation and probably plays a direct role in this process.

\section{DISCUSSION}

Sexual differentiation in the uni-cellular fungus $S$. pombe requires the breakdown of the cell wall between two cells to enable nuclear fusion and meiosis. We have used a genetic
Fig. 8. Cdc3 localizes to the projection tip during conjugation. The figure shows a series of paired panels each pair showing anti-Cdc3 immunofluorescence (top) and chromatin DAPI/phase contrast images (bottom) of the same cells. (A) In wild-type prezygotes $\mathrm{Cdc} 3$ localized to the projection tip. (B) Before (upper left) and after fusion (long cell on right) cytoplasmic dots were seen in the cytoplasm. (C) Staining with 12CA5 antibodies showed identical localization to the anti$\mathrm{Cdc} 3$ antibodies in a strain bearing a plasmid containing a fusion of $\mathrm{Cdc} 3$ and the HA epitope tag on a multi-copy plasmid but gave no signal in empty vector control strains (data not shown). The HA$\mathrm{Cdc} 3$ fusion protein localized to the projection tip, lower panel shows a DAPI/phase contrast image of the same cell. (D) Fus1-GFP and Cdc3 co-localize at the projection tip. Upper panel shows the Fus1-GFP signal, the middle panel shows anti-Cdc3 immunofluorescence and the lower panel shows DAPI/phase contrast of the same cell. Bar, $5 \mu \mathrm{m}$.
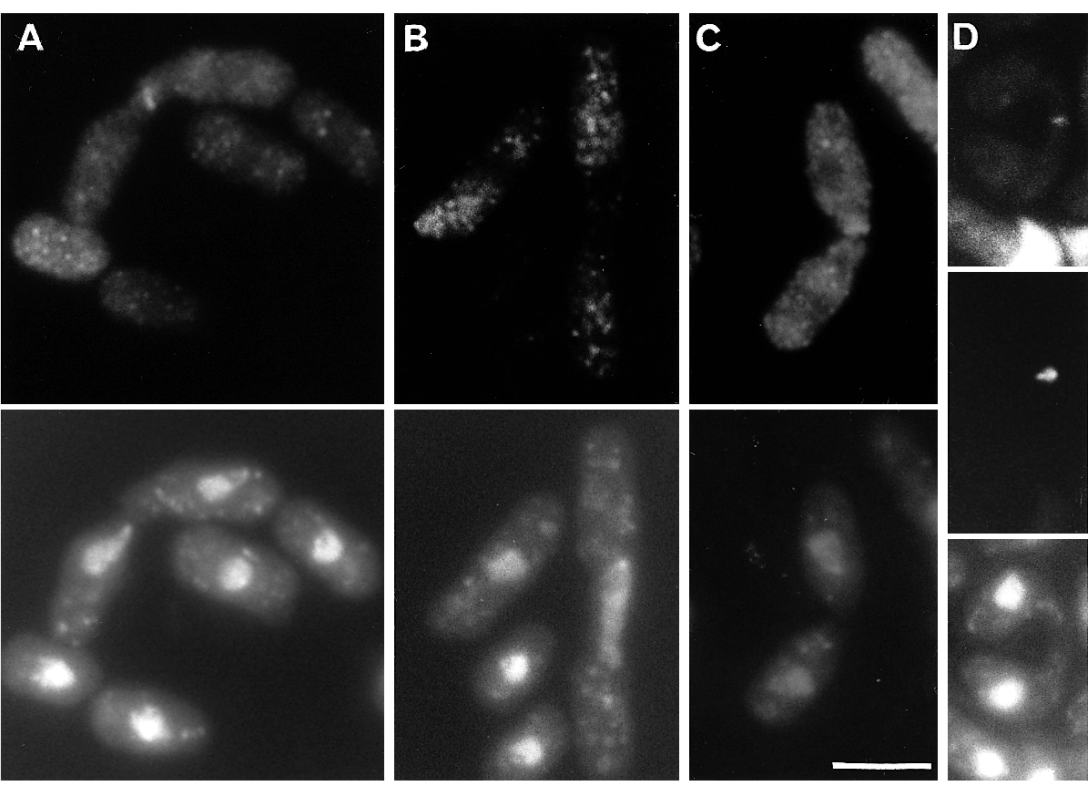
approach to identify components required for mating (Bresch et al., 1968). Here we have extended these analyses with a description of the F-actin cytoskeleton during conjugation and meiosis and have used different genetic backgrounds and the F-actin depolymerising drug LAT A to examine the functional significance of this distribution. We describe a method that synchronizes sexual differentiation. This approach enables the analysis of proteins which also function during the mitotic divisions that usually precede sexual differentiation and thus would be refractory to study in conventional mating assays.

\section{F-actin distribution and polarized cell growth}

Upon nitrogen starvation and pheromone induction fission yeast cells of the opposite mating type agglutinate and grow in a polarized fashion towards one another (Nielsen and Davey, 1995). As in mitotic growth this polarized growth is accompanied by the accumulation of F-actin patches at the growing tip (summarized in Fig. 9). Similarly, the polarized regrowth of fission yeast protoplasts is anticipated by polarized localization of actin (Kobori et al., 1989). Thus, as in fungi in general (Heath, 1990), the polarization of F-actin to the tip during conjugation is associated with tip extension. The abolition of projection tip formation and cell fusion by treatment with the actin depolymerizing drug LAT A indicates that the polarized F-actin plays a key role in these processes.

One curious feature of the actin cytoskeleton during cell fusion is the apparent absence of actin staining at the precise region of fusion where the two cell walls have been broken down. The molecular basis of this phenomenon awaits further study.

\section{Courtship in fission yeast?}

The cytoskeletal changes that accompany conjugation in fission yeast resemble those recorded in the budding yeast $S$. cerevisiae where the cortical F-actin dots have been shown to move from the main body of the cell to the point of cell extension (Waddle et al., 1996; Doyle and Botstein, 1996). In S. cerevisiae communication between two potential mating partners is termed courtship (Jackson and Hartwell, 1990). In a competition assay in which wild-type cells of one mating type had a choice of different mating partners producing different levels of pheromone, both cell types chose the mating partner, which emitted the strongest pheromone signal.

In fission yeast the $\mathrm{G}_{1}$ phase $\mathrm{F}$-actin cytoskeleton was monopolar with cortical F-actin patches at one end of the cell. This distribution switched to a bipolar one when the cells were starved of nitrogen for thirty minutes. The F-actin cytoskeleton remained bipolar for at least another half hour. Subsequently, a monopolar distribution of F-actin was seen once again as cells formed the projection tip prior to fusion (summarized in Fig. 9). This final re-polarisation of F-actin that accompanied cell fusion and the block to fusion after disruption of F-actin filaments with LAT A clearly shows that a monopolar distribution of F-actin is required for fusion. Why then does the cell generate a bipolar distribution immediately after nitrogen starvation if it is just going to make it monopolar again during conjugation? One likely explanation is that, because fission yeast generally always conjugate at their tips, a bipolar distribution doubles the chance that the molecules which will enable mating will be near a source of pheromone that is sufficiently high to allow a successful response. Furthermore,

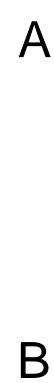

A
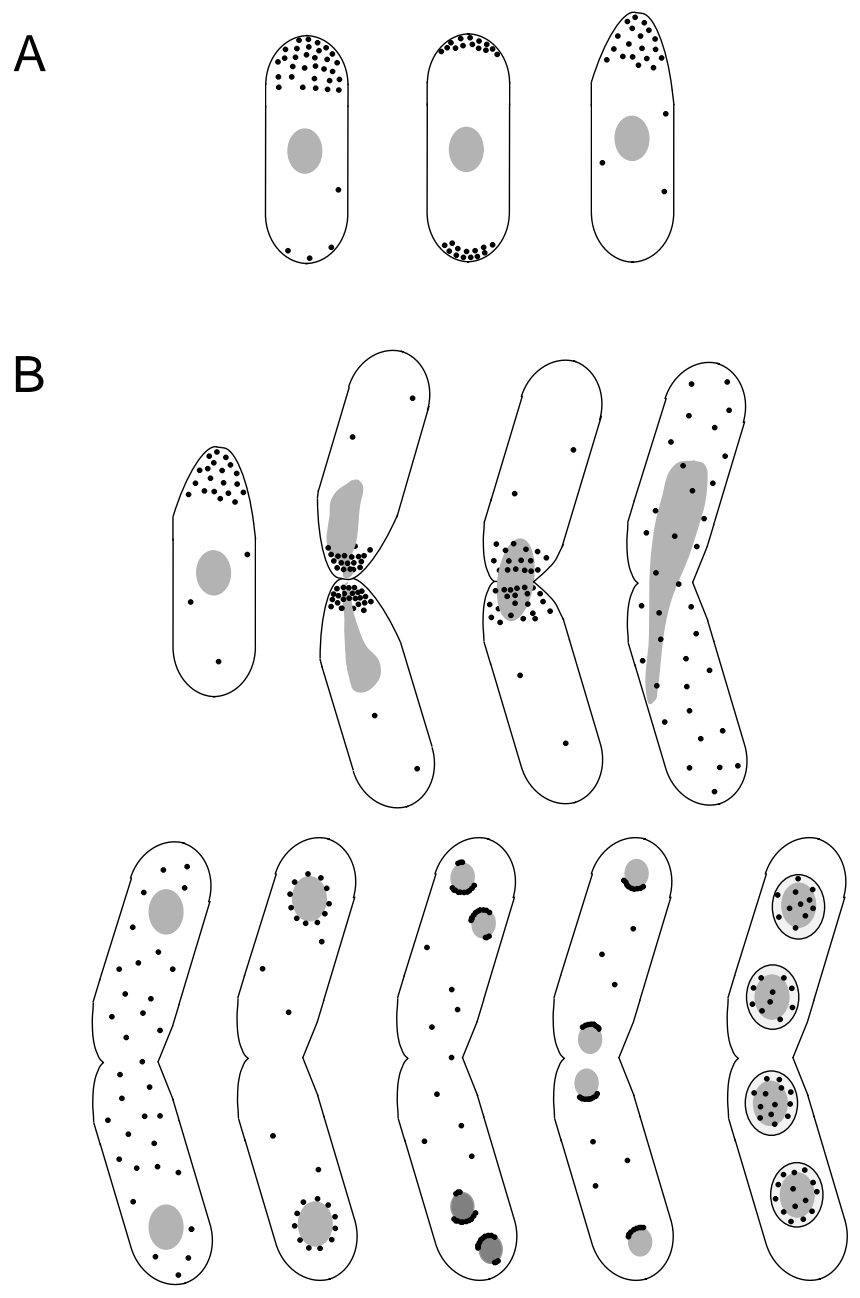

Fig. 9. Summary of F-actin localization during courtship, conjugation and meiosis in the fission yeast Schizosaccharomyces pombe. (A) During courtship nitrogen starvation induced bipolar Factin localization, which reversed to being monopolar when the projection tip formed. (B) During conjugation and meiosis F-actin localized to the fusion point and remained in this region during karyogamy. F-actin dots were subsequently scattered and remained scattered until prometaphase or metaphase of meiosis II, where they concentrated around the nuclei. During telophase F-actin was only seen on the side of the lagging nucleus which faces the partner nucleus from which it being separated. In early anaphase F-actin was also seen on the opposite side of the nucleus near the SPBs. In mature asci where the spore walls had formed F-actin concentrated within the spores.

agglutination of cells between 30 and 60 minutes after starvation indicates that they were responsive to sexual partners, however, even after 1 hour the F-actin was still bipolar. This strongly suggests that after 1 hour the cells were in a period of choice between which end to engage in mating. These two arguments strongly support a case for a courtship period in fission yeast analogous to that reported for budding yeast. As growth is from the old end immediately after division the switch to bipolar F-actin localization enhances the option of mating with sister cells, which is not favoured by F-actin localization to the old end. This is consistent with the tendency for sister cells to mate (Miyata and Miyata, 1981) 


\section{The role of F-actin in meiosis in S. pombe}

Once cell fusion and karyogamy are completed the nucleus undergoes a series of dramatic migrations along the cell axis (Chikashige et al., 1994) before committing to nuclear separation in meiosis I. Throughout this period cortical F-actin dots were randomly distributed throughout the cytoplasm (summarized in Fig. 9). At the prometaphase or metaphase of meiosis II F-actin staining appeared around the two nuclei. During anaphase of meiosis II the staining became asymmetric as a large aggregate apparently formed a cup around the side of the lagging face of the nucleus. Early on in this anaphase there was also a small $\mathrm{F}$-actin aggregate in the region around the SPB.

Electron microscopy has shown that, during metaphase of meiosis II, the outer face of the SPB differentiates and additional layers form (Tanaka and Hirata, 1982). The formation of the forespore membrane is initiated from the outermost layer of this expanded SPB. As anaphase of meiosis II proceeds the membrane extends to engulf the nucleus and gives rise to the spore wall. Because F-actin distribution around chromatin correlates with sites of cell wall deposition and spore wall deposition is blocked by disrupting actin filaments with LAT A, a major role for F-actin during meiosis in $S$. pombe is like its role during the mitotic cell cycle, to direct the deposition of cell wall material. The small aggregate associated with the SPB early in anaphase B may be associated with the uncoupling of the SPB from the forespore membrane once it has successfully initiated its synthesis. The only other situation where peri-nuclear distribution of F-actin has been reported in fission yeast is in the defective phenotype of the ben 4 mutant where abnormal rings and loops are seen around the nucleus (Marks et al., 1987). It is not clear what relation these bear to the patches that accumulate around the meiotic nuclei as they seem to be of a more filamentous nature, which has led to the suggestion that they may represent misplaced cytokinetic rings. Whilst no specific F-actin structures have been seen during meiosis in budding yeast (Doyle and Botstein, 1996; Smith et al., 1995) some reports describe peri-nuclear accumulation in other fungi (Butt and Humber, 1989; Heath 1990; ThompsonCoffe and Zickler, 1993). Interestingly the rings are seen at different stages in different fungi.

Treatment with the F-actin depolymerising drug LAT A slowed commitment to the horsetail stage and progression from karyogamy to nuclear separation in meiosis I, despite the fact that F-actin dots were apparently randomly distributed during this stage. This suggests either that the scattered F-actin dots are playing some important, though not essential function or that the drug is affecting multiple targets, such as the multiple members of the actin family, including the isoform/s required for dynein function. A full understanding of the role of actin filaments during these stages awaits further analysis.

\section{Profilin plays an essential role in mating}

Cdc3 plays an essential role in conjugation and is co-localized with the formin Fus1 at the projection tips during conjugation. The existence of the FH1 proline rich domain in formins has led to suggestions that this domain is responsible for directing the association of formins with the actin binding protein profilin in vivo because profilin will bind poly proline stretches in in vitro assays (Tanaka and Shibata, 1985). Furthermore several reports describe an in vitro interaction between profilin and the proline rich FH1 domain of formins (Chang et al., 1997; Evangelista et al., 1997; Imamura et al., 1997), and twohybrid and synthetic lethality data have been used to argue for the same interaction in vivo. In a separate study we have shown that profilin localization to the projection tip is independent of the Fus 1 proline rich FH1 domain (Petersen et al., unpublished) suggesting either that Cdc3 localization is independent of Fus 1 function or that it interacts with multiple components of a large complex, as has been suggested for the mitotic formin, $\mathrm{Cdc} 12$ (Chang et al., 1997). Cdc3 function during conjugation may differ from that during cytokinesis because F-actin localization to the tip of conjugating cells was unaffected by mutating $\mathrm{Cdc} 3$ whereas actin polymerisation into the cytokinetic ring is blocked by the same mutation.

We are very grateful to Dr Kathleen Gould for anti-Cdc3 antibodies, Dr Katherine Ayscough for Latrunculin A, Dr Chris Norbury for the cDNA library, Alan Bridge for cut12-GFP and the reviewers for constructive comments on the manuscript. This work was supported by an EMBO short term fellowship to J.P., as well as by grants from the Danish Natural Science Research Council (R.E.), the NovoNordisk Foundation (O.N.) and the Cancer Research Campaign [CRC] (I.H.). Imaging technology in Manchester was supported by the CRC and a Welcome Trust Equipment grant.

\section{REFERENCES}

Ayscough, K. R., Stryker, J., Pokala, N., Sanders M., Crews, P. and Drubin, D. G. (1997). High rates of actin filament turnover in budding yeast and roles for actin in establishment and maintenance of cell polarity revealed using the actin inhibitor Latrunculin-A. J. Cell Biol. 137, 399-416.

Balasubramanian, M. K., Hirani, B. R., Burke, J. D. and Gould, K. (1994). The Schizosaccharomyces pombe $c d c 3^{+}$gene encodes a profilin essential for cytokinesis. J. Cell Biol. 125, 1289-1301.

Bresch, C., Müller, G. and Egel, R. (1968). Genes involved in meiosis and sporulation of a yeast. Mol. Gen. Genet. 102, 301-306.

Bretscher, A., Dress, B., Harsay, E., Schott, D. and Wang, T. (1994). What are the basic functions of microfilaments? Insights from studies in budding yeast. J. Cell Biol. 126, 821-825.

Bridge, A. J., Morphew, M., Bartlett, R. and Hagan, I. M. (1998). Fission yeast Cut12 links bipolar spindle formation to mitotic control. Genes Dev. (in press).

Butt, T. M. and Humber, R. A. (1989). An immunofluorescence study of mitosis in a mite-pathogen, Neozygites sp.(Zygomycetes: Entomophthorales). Protoplasma 151, 115-123.

Carlsson, L., Nyström, L.-E., Lindberg, U., Kannan, K. K., Cid-Dresdner, H., Lövgren, S. and Jörnval, H. (1976). Crystallization of a non-muscle actin. J. Mol. Biol. 105, 353-366.

Carlsson, L., Nyström, L.-E., Sundkvist, I., Markey, F. and Lindberg, U. (1977). Actin polymerizability in influenced by profilin, a low molecular weight protein in non-muscle cells. J. Mol. Biol. 115, 465-483.

Chang, F., Drubin, D. and Nurse, P. (1997). Cdc12p, a protein required for cytokinesis in fission yeast, is a component of the cell division ring and interacts with profilin. J. Cell Biol. 137, 169-182.

Chikashige, Y., Ding, D. Q., Funabiki, H., Maraguci, T., Ashiko, S. M., Yanagida, M. and Hiraoka, Y. (1994). Telomere led premeiotic chromosome movement in fission yeast. Science 264, 270-273.

Doyle, T. and Botstein, D. (1996). Movement of yeast cortical actin cytoskeleton visualized in vivo. Proc. Nat. Acad. Sci. USA 93, 3886-3891.

Egel, R. (1971). Physiological aspects of conjugation in fission yeast. Planta 98, 89-96.

Egel, R. (1973). Commitment to meiosis in fission yeast. Mol. Gen. Genet. 121, 277-284

Egel, R., Willer, M., Kjærulff, S., Davey, J. and Nielsen, O. (1994). Assessment of pheromone production and response in fission yeast by a halo test of induced sporulation. Yeast 10, 1347-1354.

Evangelista, M., Blundell, K., Longtine, M. S., Chow, C. J., Adams, N., Pringle, J. R., Peter, M. and Boone, C. (1997). Bnilp, a yeast formin 
linking $\mathrm{Cdc} 42 \mathrm{p}$ and the actin cytoskeleton during polarized morphogenesis. Science 276, 118-122.

Griffiths, D. J., Barbet, N. C., McCready, S., Lehmann, A. R. and Carr, A. M. (1995). Fission yeast rad17: a homologue of budding yeast RAD24 that shares regions of sequence similarity with DNA polymerase accessory proteins. EMBO J. 14, 5812-5823.

Gutz, H., Heslot, H., Leupold, U. and Loprieno, N. (1974). Schizosaccharomyces pombe. In Handbook of Genetics, vol 1 (ed. R. C. King), pp. 395-466. Plenum Press, New York.

Haarer, B. K., Lillie, S. H., Adams, A. E. M., Magdolen, V., Bandlow, W. and Brown, S. S. (1990). Purification of profilin from Saccharomyces cerevisiae and analysis of profilin-deficient cells. J. Cell Biol. 110, 105114.

Hagan, I. M. and Hyams, J. S. (1988). The use of cell division cycle mutants to investigate the control of microtubule distribution in the fission yeast Schizosaccharomyces pombe. J. Cell Sci. 89, 343-357.

Hagan, I. and Yanagida, M. (1995). The product of the spindle formation gene, sadl $^{+}$associates with the fission yeast spindle pole body and is essential for viability. J. Cell Biol. 129, 1033-1047.

Hanahan, D. (1985). Techniques for transformation of E. coli. In DNA Cloning, a Practical Approach (ed. D. M. Glover), pp. 109-135. IRL Press, New York.

Heath, I. B. (1990). The roles of actin in tip growth of fungi. Int. Rev. Cytol. 123, $95-127$.

Imamura, H., Tanaka, K., Hihara, T., Umikawa, M., Kamei, T., Takahashi, K., Sasaki, T. and Takai, Y. (1997). Bnilp and Bnrlp: downstream targets of the Rho family small G-proteins which interact with profilin and regulate actin cytoskeleton in Saccharomyces cerevisiae. EMBO J. 16, 745-2755.

Jackson, C. L. and Hartwell, L. H. (1990). Courtship in Saccharomyces cerevisiae: both cell types choose mating partners by responding to the strongest pheromone signal. Cell 63, 1039-1051.

Jansen, R.-P., Dowzer, C., Michaelis, C., Galova, M. and Nasmyth, K. (1996). Mother cell-specific $H O$ expression in budding yeast depends on the unconventional myosin Myo4p and other cytoplasmic proteins. Cell 84, 687697.

Kobori, H., Yamada, N., Taki, A. and Osumi, M. (1989). Actin is associated with the formation of the cell wall in reverting protoplast of the fission yeast Schizosaccharomyces pombe. J. Cell Sci. 94, 635-646.

Kocher, T. D., Thomas, W. K., Meyer, A., Edwards, S. V., Paabo, S., Villablanca, F. X. and Wilson, A. C. (1989). Dynamic of mitochondrial DNA evolution in animals: amplification and sequencing with conserved primers. Proc. Nat. Acad. Sci. USA 86, 6196-6200.

Kilmartin, J. V. and Adams, A. E. M. (1984). Structural rearrangements of tubulin and actin during the cell cycle of the yeast Saccharomyces cerevisiae. J. Cell Biol. 98, 922-939.

Marks, J. and Hyams, J. S. (1985). Localization of F-actin through the cell division cycle of Schizosaccharomyces pombe. Eur. J. Cell. Biol. 39, 27-32.

Marks, J., Hagan, I. M. and Hyams, J. S. (1987). Spatial association of Factin with growth polarity and septation in the fission yeast Schizosaccharomyces pombe. In Spatial organization in eukaryotic microbes (ed. R. K. Poole and A. P. C. Trinci), pp. 119-135. IRL Press, Oxford.

Marks, J., Fankhauser, C., Reymond, A. and Simanis, V. (1992). Cytoskeletal and DNA structure abnormalities result from bypass of requirement of the $c d c 10$ start gene in fission yeast Schizosaccharomyces pombe. J. Cell Sci. 100, 517-528.

Meloche, S., Pagès, G. and Pouysségur, J. (1992). Functional expression and growth factor activation of an epitope-tagged $\mathrm{p} 44$ mitogen-activated protein kinase p44 ${ }^{\mathrm{mapk}}$. Mol. Biol. Cell 3, 63-71.

Mertins, P. and Gallwitz, D. (1987). A single intronless actin gene in the fission yeast Schizosaccharomyces pombe - nucleotide sequence and transcripts formed in homologous and heterologous yeast. Nucl. Acids Res. 15, 7369-7379.

Mitchison, J. M. and Nurse, P. (1985). Growth in cell length in fission yeast. J. Cell Sci. 75, 357-376.

Miyata, H. and Miyata, M. (1981). Mode of conjugation in homothallic cells of Schizosaccharomyces pombe. J. Gen. Appl. Microbiol. 27, 365-371.

Moreno, S., Klar, A. and Nurse, P. (1991). An introduction to molecular genetics analysis of the fission yeast Schizosaccharomyces pombe. Meth. Enzymol. 194, 795-823.

Mullins, R. D., Kelleher, J. F. and Pollard, T. D. (1996). Actin like actin? Trends Cell Biol. 6, 208-211.

Nielsen, O. and Egel, R. (1990). The pat1 protein kinase controls transcription of the mating-type genes in fission yeast. EMBO J. 9, 1401-1406

Nielsen, O., Davey, J. and Egel, R. (1992). The ras1 function of Schizosaccharomyces pombe mediates pheromone-induced transcription. EMBO J. 11, 1391-1395.

Nielsen, O. and Davey, J. (1995). Pheromone communication in the fission yeast Schizosaccharomyces pombe. Semin. Cell Biol. 6, 65-104.

Nurse, P. and Bissett, Y. (1981). Gene required in $\mathrm{G}_{1}$ for commitment to cell cycle and in $\mathrm{G}_{2}$ for control of mitosis in fission yeast. Nature 292, 558-560.

Nurse, P., Thuriaux, P. and Nasmyth, K. (1976). Genetic control of the cell division cycle in the fission Schizosaccharomyces pombe. Mol. Gen. Genet. 146, 167-178.

Petersen, J., Weilguny, D., Egel, R. and Nielsen, O. (1995). Characterization of fus 1 of Schizosaccharomyces pombe: A developmentally controlled function needed for conjugation. Mol. Cell. Biol. 15, 3697-3707.

Robinow, C. F. (1977). The number of chromosomes in Schizosaccharomyces pombe: light microscopy of stained preparations. Genetics 87, 491-497.

Robinow, C. F. and Hyams, J. S. (1989). General cytology of fission yeast. In The Molecular Biology of the Fission Yeast (ed. A. Nasin, P. G. Young and B. F Johnson), pp. 273-331. Academic Press New York.

Sambrook, J., Fritsch, E. F. and Maniatis, T. (1989). Molecular Cloning: a Laboratory Manual, 2nd edn. Cold Spring Harbor Laboratory Press, Cold Spring Harbor, New York.

Smith, G. M., Simon, V. R., O'Sullivan, H. and Pon, L. A. (1995) Organelle-cytoskeletal interactions: Actin mutations inhibit meiosisdependent mitochondrial rearrangement in the budding yeast Saccharomyces cerevisiae. Mol. Biol. Cell 6, 1381-1396.

Sohn, R. H. and Goldschmidt-Clermont, P. J. (1994). Profilin: at the crossroad of signal transduction and the actin cytoskeleton. BioEssays 16, 465-472.

Tanaka, K. and Hirata, A. (1982). Ascospore development in the fission yeast Schizosaccharomyces pombe. J. Cell Sci. 56, 263-279.

Tanaka, M. and Shibata, H. (1985). Poly-(L-proline)-binding proteins from chick embryos are a profilin and a profilactin. Eur. J. Biochem. 151, 291-297.

Thompson-Coffe, C. and Zickler, D. (1993). Cytoskeletal interactions in the acsus development and sporulation of Sordaria macrospora. J. Cell Sci. 104, 883-898.

Waddle, J. A., Karpova, T. S., Waterston, R. H. and Cooper, J. A. (1996). Movement of cortical actin patches in yeast. J. Cell Biol. 132, 861-870.

Wilson, I. A., Niman, H. L., Houghten, R. A., Cherenson, A. R., Connolly, M. L. and Lerner, A. R. (1984). The structure of an antigen determinant in a protein. Cell 37, 767-778. 